\title{
Kernos
}

Revue internationale et pluridisciplinaire de religion grecque antique

16 | 2003

Varia

\section{J.-P. MAYELE ILO, Statut mythique et scientifique de la gémellité}

\section{Véronique Dasen}

URL : http://journals.openedition.org/kernos/856

DOI : $10.4000 /$ kernos.856

ISSN : 2034-7871

\section{Éditeur}

Centre international d'étude de la religion grecque antique

\section{Édition imprimée}

Date de publication : 1 janvier 2003

Pagination : 386-387

ISSN : 0776-3824

Référence électronique

Véronique Dasen, «J.-P. mAYele ILo, Statut mythique et scientifique de la gémellité », Kernos [En ligne], 16 | 2003, mis en ligne le 14 avril 2011, consulté le 22 septembre 2020. URL : http:// journals.openedition.org/kernos/856 ; DOI : https://doi.org/10.4000/kernos.856 
traduisent leur aspiration à la divinisation en commandant leur portrait (ou ceux de leurs proches) dans ce matériau hors du commun qui évoque le corps impérissable des dieux.

Bien illustré, l'ouvrage est accompagné d'un catalogue des fragments de statues retrouvées, d'un choix de textes relatifs aux ouvres disparues (version originale et traduction), d'une bibliographie très complète et de deux appendices (une liste des statues mentionnées dans les sources littéraires et épigraphiques par site, sujet et période, ainsi qu'une discussion de la description par Pausanias de l'Athéna Areia acrolithe de Platées).

Véronique Dasen

(Université de Friboug)

Jean-Pierre Maýele Ilo, Statut mythique et scientifique de la gémellité. Essai sur la dualité, Bruxelles, Ousia, 2001. 1 vol. $14 \times 20 \mathrm{~cm}, 605$ p. (Mythes et religions). ISBN : 2-87060-083-6.

Le phénomène gémellaire occupe une place importante dans la pensée mythique universelle. Si sa fonction au sein du monde indo-européen a fait l'objet de nombreux travaux, une synthèse sur son rôle en Afrique noire manque encore. Tout en relevant cette absence, l'A. n'y répond pas de manière directe. La gémellité lui sert de moyen pour aborder de manière plus générale les rapports entre philosophie, mythe et science afin de démontrer d'une part que la fonction du mythe correspond à une démarche de la pensée qui ne diffère pas de celle de la pensée scientifique, d'autre part que " mythe et gémellité sont deux versions différentes mais superposables d'une seule et même réalité fondamentale, celle de la pensée totale" (p. 31). Afin de clarifier son propos, il crée un néologisme, la dyosynchrogénésie, pour distinguer lá réalité biologique des jumeaux de la gémellité qu'il traite comme une pensée, une logique de la « dualitude ».

L'A. s'appuie sur les réflexions de mythologues classiques pour tenter de saisir le fonctionnement de la pensée archä̈que d'Afrique noire et ses correspondances avec la pensée scientifique. Dans le $1^{\text {er }}$ chapitre, partant des travaux de M. Detienne, l'A. démontre que le mythe doit être défini autrement que comme une narration orale ou écrite, centrée sur la généalogie. Sa nature transcendentale et son universalité sont liées à sa structure, qui articule le monde visible et le monde invisible. Il est d'abord découverte de l'imbrication de ces deux niveaux, humain et divin, qui échappe à l'expérience sensorielle directe de l'homme. Le $2^{e}$ chapitre examine la notion de "vérité » ou d'« efficacité » du mythe, pour remettre en question sa dimension étiologique. Synonyme d'expérience vécue, universelle, entre l'homme et une Présence, il fonctionne comme une métaphore. Cette dimension métaphorique explique l'importance des jumeaux dans la pensée archä̈que d'Afrique noire, à laquelle l'A. consacre les $3^{\mathrm{e}}$ et $4^{\mathrm{e}}$ chapitres, mais en adoptant un biais particulier. Il confronte en les détaillant la notion de causalité naturelle ou surnaturelle dans le discours scientifique (facteurs génétiques, biochimiques, environnementaux) et clans la pensée archaïque d'Afrique noire (Dogon, Luba, Baluba, Swazi...) pour montrer que mythe et science présentent des hypothèses qui articulent les mêmes types de facteurs, connus et inconnus (le visible et l'invisible), déterminés et indéterminés (déterminisme biologique et le milieu au sens large). Dans le discours scientifique, l'élément déclencheur échappe encore, faute d'un "supplément d'âme". Dans la pensée afroarchaîque, la gémellité est définie par son extranéité. Une entité invisible décide de la grossesse de la femme et détermine la naissance de jumeaux qui représente une «ancestrophanie ", l'avènement de l'invisible dans le monde visible.

L'A. conclut par une mise en parallèle des réalités culturelles afro-archä̈ques avec celles du monde classique et indo-européen, mais sans vraiment développer la comparaison. Son souci reste de démontrer qu'il existe un rapport entre le phénomène gémellaire et le mythe qui n'est pas de l'ordre du concept mais qui relève de leur structure et de leur équilibre dynamique. La gémellité est une pensée en acte, fonctionnant comme une métaphore, c'est-à-dire comme un mythe.

Dépourvu d'index, cet ouvrage au titre prometteur n'expose pas la synthèse attendue sur les différentes fonctions symboliques de la gémellité en Afrique Noire. Réflexion philosophique, il consiste en la démonstration circonstanciée d'une hypothèse (la gémel- 
lité est synonyme de mythe et témoigne d'une Présence) qui demande au lecteur une ténacité certaine pour la suivre dans tous les détails.

Véronique Dasen

(Université de Fribourg)

Pier Franco BeAtrice, Anonymi Monophysitae Theosophia. An Attempt at Reconstruction, Leiden, Brill, 2001, LXXI + 140 p. (Suppl. to Vigiliae Christianae, 56). ISBN : 90-04-11798-9.

En 1995, Hartmut Erbse a fait paraître un livre intitulé Theosophorum Graecorum Fragmenta (Bibliotbeca Scriptorum Graecorum et Romanorum Teubneriana), Stuttgart/ Leipzig (dorénavant Erbse, Fragmenta); il s'agit de l'édition critique de différentes recensions de textes 'théosophiques' grecs, tant païens que chrétiens (sentences, oracles, apophtegmes et d'autres fragments); cette édition revoit celle que le même auteur a établie dans ses Fragmente griechischer Theosopbien (Hamburger Arbeiten zur Altertumswissenschaft 4), Hamburg, 1941 (dorénavant Erbse, Fragmente). À peine cinq années sont passées, et voici une nouvelle édition, préparée par Pier Franco Beatrice. La première remarque qui s'impose ici, est la suivante : encore une édition du même texte! Il n'y a donc rien d'étonnant à ce que Beatrice consacre une partie de son introduction à montrer l'importance de son travail. Tandis que Erbse a juxtaposé les différentes recensions et les multiples fragments, Beatrice essaie de reconstruire le texte original de l'œuvre appelée $\Theta \epsilon о \sigma o \phi l a$ et divisée en quatre livres; les résultats de reconstruction auxquels Beatrice a abouti, nous semblent convaincants. Probante est également la datation et l'attribution du texte qu'il propose: il l'attribue à Sévère, patriarche monophysite d'Antioche de 512 à 518; donc l'ouvrage revient au début du $\mathrm{vI}^{\mathrm{e}}$ siècle et s'inspire de l'hérésie monophysite. Une brève étude de la tradition manuscrite et des éditions imprimées est suivie du texte critique de l'œuvre; un index locorum Sacrae Scripturae et un index fontium in textu laudatorum facilitent la consultation du livre (malheureusement il n'y a ni index des incipit des fragments, ni index graecitatis).

Nous voudrions formuler deux remarques générales sur le texte établi. Tout d'abord, nous avons constaté qu'il y a un grand nombre de petites fautes qui entament vraiment la valeur du texte grec (par exemple : p. 3, 1. 17 ËTous pour ËTous; p. 8, 1. 17 пa

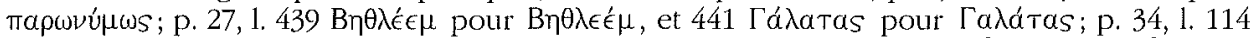

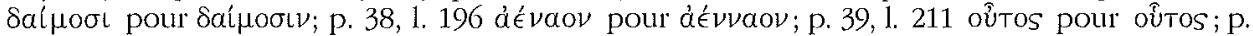

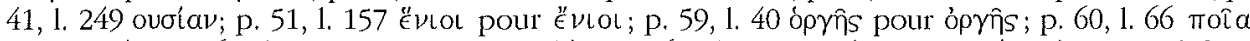

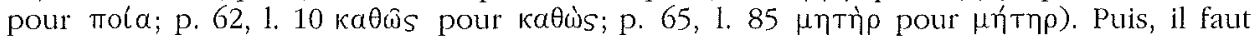
également déplorer que pas mal de références bibliques manquent dans l'apparat des sources (par exemple: p. 41, 1. 253: Act. 17, 28; p. 59, 1. 30-31: Lc. 18, 27; p. 65, 1. 71: Lc. 10, 19; p. 67, 1. 114-115: Mt. 10, 21).

En guise de conclusion de notre compte rendu, nous signalons un témoin resté inaperçu jusqu'ici. Il s'agit de l'Athous, Iviron 386 dont nous publierons une description très détaillée dans un livre à parâtre consacré à la tradition manuscrite de Maxime le Confesseur, théologien byzantin du $v{ }^{\mathrm{e}}$ siècle. Le manuscrit semble dater de la première partie du $x{ }^{e}{ }^{e}$ siècle; aucune note ne nous renseigne ni sur l'endroit de confection du manuscrit, ni sur son histoire postérieure; le volume, sauf pour les folios initiaux contenant un pinax et pour deux folios non numérotés qu'on trouve entre les actuels folios 125 et 127, revient à un seul scribe resté anonyme. La plus grande partie, bien connue des érudits, est consacrée à la querelle hésychaste. Mais il y a d'autres sections. Ainsi, les f. 169$193^{\mathrm{V}}$ contiennent une collection assez disparate de petits textes. Le texte qui nous intéresse ici est précédé immédiatement d'un petit florilège, qui se compose principalement de fragments pseudo-athanasiens (f. 170-174), et est suivi de la collection athonite des proverbes de Zenobius (f. $176^{\mathrm{V}}-190^{\mathrm{V}}$ ).

Ce sont donc les f. $174^{\mathrm{V}}-176$ qui contiennent une partie de la $\Theta \epsilon o \sigma o \phi i a$; nous en avons fait la collation, et voici nos constatations. Nous avons comparé le texte du manuscrit de la Sainte Montagne avec l'édition de Erbse; en effet, il est plus facile d'utiliser cette édition, car celle-ci donne l'édition de toutes les recensions de l'ceuvre.

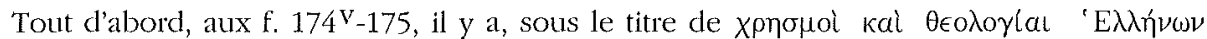
$\phi(\lambda o \sigma o ́ \phi \omega \nu$, une partie de la recension $\chi$. En voici l'inventaire: $\chi 9$ (le titre de ce fragment 